\title{
Avaliação dos coeficientes de digestibilidade dos aminoácidos do grão de trigo e seus subprodutos para frangos de corte utilizando diferentes metodologias
}

[Evaluation of the digestibilty coefficients of amino acids of wheat grain and its by-products for broiler chickens, using different methodologies]

\author{
F.M.O. Borges ${ }^{1}$, H.S. Rostagno ${ }^{2}$, C.E.P. Saad ${ }^{3}$, N.M. Rodriguez ${ }^{4}$, \\ E.A. Teixeira ${ }^{5}$, L.B. Lara $^{5}$, W.S. Mendes ${ }^{5}$, V.L. Araújo ${ }^{5}$ \\ ${ }^{1}$ Departamento de Zootecnia da Universidade Federal de Lavras \\ Caixa Postal 37 \\ 37200-000 - Lavras, MG \\ ${ }^{2}$ Universidade Federal de Viçosa \\ ${ }^{3}$ Zootecnista \\ ${ }^{4}$ Escola de Veterinária da Universidade Federal de Minas Gerais \\ ${ }^{5}$ Médico Veterinário
}

Recebido para publicação em 13 de fevereiro de 2003

Recebido para publicação, após modificações, em 22 de setembro de 2003

E-mail: borgesvet@ufla.br

\section{RESUMO}

Realizou-se um experimento com frangos de corte para avaliação dos coeficientes de digestibilidade dos aminoácidos ((coeficiente de digestibilidade aparente (CDAaa) e coeficiente de digestibilidade verdadeira (CDVaa)) comparando quatro metodologias de estimação. O delineamento experimental foi inteiramente ao acaso, em esquema fatorial $4 \times 8$ (tratamentos $\times$ alimentos) com quatro repetições. Sete alimentos oriundos do trigo e uma ração-referência foram utilizados nos quatro tratamentos: trigo integral moído, gérmen de trigo, farinha de trigo clara, farinha de trigo escura, farelo de trigo para uso humano, farelo de trigo para uso animal e farelo de trigo grosso. Os dois primeiros tratamentos foram baseados na metodologia tradicional de consumo a vontade, diferindo apenas quanto à idade das aves (13 e 39 dias, respectivamente). Os alimentos testados substituíram $40 \%$ da ração-referência. Foram registradas as quantidades ingeridas e coletadas as excretas totais. Nos tratamentos 3 e 4 utilizou-se o método da alimentação forçada. Neles as aves, submetidas a jejum de 30 horas, foram forçadas a ingerir 25 gramas de alimento puro de uma única vez, utilizando o alimento puro, sem níveis de substituição. Em todos os tratamentos uma parcela foi deixada em jejum, para determinação das perdas metabólica e endógena. O tratamento 4 foi realizado com aves cecectomizadas. Nos alimentos e nas excretas foram analisadas matéria seca, proteína bruta e aminoácidos. O método tradicional apresentou maiores valores de CDAaa, quando comparado ao método de alimentação forçada. Na farinha de trigo clara e farinha de trigo escura esses valores foram negativos, mostrando que a perda endógena foi maior que a ingestão de aminoácidos. Os alimentos alto em fibra (farelo de trigo para uso humano, farelo de trigo para uso animal e farelo de trigo grosso) apresentaram os menores valores de CDAaa e CDVaa nos tratamentos com metodologia tradicional e nos de alimentação forçada, confirmando a interferência da fibra sobre a digestibilidade dos aminoácidos. Quanto aos valores de CDVaa, as diferenças foram menores quando se compararam os tratamentos pelo método tradicional e de alimentação forçada, entretanto, as aves cecectomizadas apresentaram menor CDAaa, devido à maior perda metabólica e endógena.

Palavras-chave: frango, aminoácido digestível, cecectomia

\section{ABSTRACT}


One experiment was run with broiler chickens to evaluate the coefficients of digestibility of amino acids (coefficients of apparent digestibility of amino acids - CDAaa and coefficients of true digestibility of amino acids - CDVaa) using wheat grain and some of its by-products, comparing four methodologies. The experiment was a completely randomized factorial design $4 \times 8$ (treatments $\times$ feedstuffs) with four replicates. Seven wheat grain by-products were used: wheat grain, wheat germ, white wheat flour, dark wheat flour, wheat bran for human use, wheat bran for animal use and rough wheat bran. Treatments 1 and 2 were the traditional methodology with ad libitum feed consumption, just differing in the age of the birds, 13 and 39 day-old, respectively. The feed tested substituted $40 \%$ of a reference diet. Feed intake and total excreta were recorded. The forced feeding method was used (Sibbald, 1976) for treatments 3 and 4. The birds were fasted 30 hours and forced fed $25 \mathrm{~g}$ of the feed ingredients. In all the experiments a treatment was included with fasting birds to determine metabolic and endogenous losses. In method four caecectomized birds were used. Feeds and excreta were analyzed for dry matter, protein and amino acids. The traditional methods showed higher CDAaa values when compared to the forced feeding methods. For white wheat flour and dark wheat flour these values were negative, showing that endogenous losses were larger than amino acid intake. High fiber feedstuffs (wheat bran for human use, wheat bran for animal use and rough wheat bran) showed the lowest CDAaa and CDVaa values in the traditional and forced fed methods, confirming the interference of crude fiber on amino acid digestibility. In regard to the CDVaa, differences were smaller when compared the traditional and the forced fed methods, however, caecectomized birds showed lower CDAaa, due to high metabolic and endogenous losses.

Keywords: bird, digestible amino acid, caecectomy

\section{INTRODUÇÃO}

Os cálculos de necessidades de alimentos para as aves e o planejamento das dietas têm-se baseado em tabelas de requisitos, nas quais são expressas tanto as necessidades dos animais como o valor nutritivo dos alimentos. Na nutrição não é importante estimar os requisitos das aves em termos de necessidade metabólica se não há informação do valor potencial do alimento. Dessa forma, além de avaliar os requisitos de proteína e aminoácidos das aves é necessário quantificar e qualificar o nível de nutrientes nos alimentos, de modo a diminuir os erros na formulação de dietas (Mejía, Ferreira, 1996).

Segundo Bellaver (1996), é grande o número de métodos usados para a determinação da qualidade das proteínas e da disponibilidade dos aminoácidos. O termo disponibilidade muitas vezes tem sido utilizado como digestibilidade. Define-se disponibilidade como a quantidade de aminoácido no alimento que pode ser utilizada biologicamente pela ave e digestibilidade como a quantidade de aminoácidos absorvida pelo trato digestivo.

Sibbald (1987) esclareceu a relação entre os dois termos ao assinalar que a absorção de nutrientes no trato digestivo é pré-requisito para a sua utilização, mas não demonstra que tenha que existir disponibilidade. Alguns aminoácidos presentes por exemplo nas proteínas danificadas pelo calor podem ser absorvidos e excretados na urina, o que, em conseqüência, indicaria que eles não se encontravam disponíveis para serem utilizados no metabolismo animal. O mesmo autor considerou que a utilização de um nutriente absorvido no trato digestivo é evidência de sua disponibilidade, porém, a excreção não prova a sua perda.

Segundo Engster et al. (1985), diferentes métodos de determinação têm sido utilizados para avaliar os valores de digestibilidade de aminoácidos para aves, incluindo ensaios de crescimento, ensaios químicos e microbiológicos, medição de aminoácidos do sangue e tratamentos de balanço nos quais aminoácidos do íleo ou das fezes são mensurados. Cada um desses trabalhos apresenta méritos e limitações e podem resultar em valores diferentes.

O bioensaio feito por Sibbald (1976) para estimativa da energia metabolizável verdadeira pode-se prestar à determinação dos aminoácidos digestíveis. Likuski e Dorell (1978) citam que esse método pode ser 
interessante pois é rápido e de baixo custo. Entretanto, ele apresenta limitações. Engster et al. (1985) mencionam que um dos pontos falhos seria o desconhecimento da contribuição dos aminoácidos microbianos na excreta, além de não se saber ao certo se os aminoácidos urinário e endógeno seriam os mesmos nas aves em jejum e alimentadas com os alimentos-teste. Como vantagem pode-se mencionar que, em adição à medição de cada aminoácido simultaneamente, é possível determinar ao mesmo tempo os valores de energia metabolizável verdadeira corrigida pelo balanço de nitrogênio (EMVn).

Segundo Albino (1991), um dos fatores que interferem na digestibilidade dos aminoácidos é a atividade da microflora do intestino. $\mathrm{O}$ autor questiona se a microflora intestinal pode modificar a composição da excreta por degradação dos aminoácidos ou síntese de compostos nitrogenados que serão excretados. Uma alternativa, segundo Crissey e Thomas (1987), seria a utilização de galos cecectomizados uma vez que o ceco é o local de maior atividade microbiana.

Segundo Albino (1991), o conhecimento da digestibilidade dos aminoácidos dos alimentos possibilitaria utilizar com mais eficiência alimentos alternativos na formulação de rações.

Os objetivos deste trabalho foram avaliar a digestibilidade aparente e verdadeira dos aminoácidos essenciais e não essenciais de oito alimentos (ração-referência e sete subprodutos do trigo), comparando as metodologias tradicional e de alimentação forçada para pintos e frangos, e avaliar os efeitos da cecectomia na digestibilidade aparente e verdadeira dos aminoácidos, pela metodologia de alimentação forçada, utilizando aves normais e cecectomizadas.

\section{MATERIAL E MÉTODOS}

Para a determinação dos aminoácidos digestíveis dos alimentos (RR= ração-referência, TIM=trigo integral moído, GT= germe de trigo, FTC= farinha de trigo clara, FTE= farinha de trigo escura, FTH= farelo de trigo para uso humano, FTA= farelo de trigo para uso animal, FTG= farelo de trigo grosso), utilizaram-se as excretas obtidas em quatro tratamentos com aves, usadas anteriormente para determinação da energia metabolizável (Borges et al., 2003). Alimentos e tratamentos, delineamento experimental, metodologia empregada e modelo estatístico utilizados foram descritos em Borges et al. (2003), com exceção de alguns procedimentos detalhados na seqüência.

A cecectomia foi realizada segundo a técnica descrita por Pupa et al. (1998). As análises de aminoácidos foram feitas por hidrólise ácida e leitura por cromatografia líquida de alta eficiência (CLAE) em coluna de troca iônica, com reação pós-coluna com ortoftaloleído (OPA) nos alimentos puros, ração-referência e excreta, utilizando-se cromatógrafo de fase líquida Shimatzu - 10A, com detector de fluorescência (Motter, 1991).

Nos alimentos puros foram analisados os aminoácidos essenciais valina (VAL), metionina (MET), isoleucina (ISO), leucina (LEU), treonina (TRE), fenilalanina (FEN), histidina (HIST), lisina (LIS) e arginina (ARG), e os não essenciais aspartato (ASP), tirosina (TIR), serina (SER), glutamina (GLU), prolina (PRO), glicina (GLI), alanina (ALA) e cistina (CIS).

Embora tenham sido analisados os teores de cistina, glicina e arginina nos alimentos, não se determinaram suas digestibilidade. Também não foi determinado o coeficiente de digestibilidade do triptofano. As razões técnicas que inviabilizaram esses resultados são descritas a seguir.

Os aminoácidos arginina e glicina chegaram a ser dosados por cromatografia líquida, entretanto a arginina apresentou problemas nos picos de detecção do aparelho, optando-se pela eliminação dos dados. A glicina apresentou ótimos picos de deteç̧ão, no entanto os dados foram eliminados em razão da presença de ácido úrico na excreta das aves, o qual apresenta uma molécula de glicina em sua estrutura, liberada quando o ácido úrico é hidrolisado. Com relação à cistina, os valores não foram determinados pelo fato desse aminoácido poder sofrer grande destruição durante a hidrólise ácida. O mesmo pode ocorrer com a 


\section{Borges et al.}

metionina, porem em menor quantidade (Albino, 1991). Dessa forma, uma vez que a destruição da metionina é pequena e presumindo-se que essa destruição ocorra também nas análises dos alimentos, optou-se por manter esses dados, aspecto já sugerido por Muztar e Slinger (1980), Kessler et al. (1981) e Albino (1991).

As análises estatísticas foram feitas no laboratório de computação científica da Universidade Federal de Minas Gerais, em um computador IBM 4341.

O delineamento experimental foi inteiramente ao acaso, em esquema fatorial $4 \times 8$ (tratamentos $\times$ alimentos) com quatro repetições. As médias foram comparadas pelo teste SNK e referiam-se aos aminoácidos digestíveis.

Nos tratamentos com a metodologia de Sibbald (tratamentos 3 e 4) foram determinadas diretamente a ingestão e excreção de cada aminoácido por alimento. Como se conhecia a porcentagem de cada aminoácido, os coeficientes de digestibilidade foram calculados.

Nos tratamentos com a metodologia tradicional (tratamentos 1 e 2) as quantidades conhecidas eram da ração-teste consumida, do total excretado e da proporção de substituição do alimento na ração-referência, aspectos já destacados por Borges et al. (2003). Além disso, foram determinadas as porcentagens de cada um dos aminoácidos nas rações-teste e na excreta. Os cálculos dos coeficientes de aminoácidos digestíveis aparente e verdadeiro dos alimentos, utilizando a metodologia tradicional de alimentação, foram semelhantes aos utilizados por Borges et al. (2003) para energia metabolizável.

\section{RESULTADOS E DISCUSSÃO}

A composição de aminoácidos essenciais e não essenciais da ração-referência dos alimentos são apresentadas nas Tab. 1 e 2, respectivamente. O germe de trigo apresentou os maiores valores de aminoácidos essenciais. As farinhas de trigo (FTC e FTE) apresentaram menores valores para todos os aminoácidos essenciais. Os farelos de trigo (FTH, FTA e FTG) mostraram valores de aminoácidos menores do que os valores de aminoácidos para esses alimentos descritos nas tabelas de Rostagno et al. (1985).

Tabela 1. Composição em aminoácidos essenciais (\%/MS) dos alimentos

\begin{tabular}{lcccccccc}
\hline \multirow{2}{*}{ Aminoácido } & \multicolumn{7}{c}{ Alimento $^{1}$} \\
\cline { 2 - 8 } & RR & TIM & GT & FTC & FTE & FTH & FTA & FTG \\
\hline Valina & 0,99 & 0,66 & 0,86 & 0,43 & 0,36 & 0,75 & 0,55 & 0,67 \\
Metionina & 0,48 & 0,23 & 0,36 & 0,14 & 0,15 & 0,24 & 0,17 & 0,21 \\
Isoleucina & 0,89 & 0,52 & 1,00 & 0,76 & 0,24 & 1,07 & 0,40 & 1,02 \\
Leucina & 2,04 & 1,16 & 1,77 & 0,80 & 0,85 & 1,10 & 0,86 & 1,07 \\
Treonina & 0,78 & 0,39 & 0,74 & 0,20 & 0,31 & 0,44 & 0,34 & 0,44 \\
Fenilalanina & 1,31 & 0,93 & 1,26 & 0,63 & 0,65 & 0,81 & 0,68 & 0,80 \\
Histidina & 0,87 & 0,56 & 1,21 & 0,34 & 0,41 & 0,65 & 0,52 & 0,59 \\
Lisina & 1,62 & 0,56 & 2,32 & 0,32 & 0,45 & 0,99 & 0,74 & 0,72 \\
Arginina & 2,35 & 1,06 & 2,99 & 0,53 & 0,76 & 1,85 & 1,24 & 1,51 \\
\hline
\end{tabular}

1: RR= ração-referência, TIM= trigo integral moído, GT= germe de trigo, FTC= farinha de trigo clara, FTE= farinha de trigo escura, FTH= farelo de trigo para uso humano, FTA= farelo de trigo para uso animal, FTG= farelo de trigo grosso. 
Avaliação dos coeficientes de digestibilidade...

Tabela 2. Composição em aminoácidos não essenciais (\%/MS) dos alimentos

\begin{tabular}{lllllllll}
\hline \multirow{2}{*}{ Aminoácido } & \multicolumn{6}{c}{ Alimento $^{1}$} \\
\cline { 2 - 7 } & RR & TIM & GT & FTC & FTE & FTH & FTA & FTG \\
\hline Aspartato & 2,55 & 0,81 & 2,94 & 0,47 & 0,7 & 1,26 & 0,96 & 1,02 \\
Tirosina & 0,94 & 0,52 & 1,13 & 0,33 & 0,37 & 0,63 & 0,49 & 0,54 \\
Serina & 1,26 & 0,86 & 1,46 & 0,59 & 0,71 & 0,81 & 0,64 & 0,81 \\
Glutamina & 5,11 & 7,05 & 5,44 & 5,33 & 5,3 & 3,86 & 3,28 & 5,19 \\
Prolina O & 1,64 & 2,25 & 1,63 & 1,69 & 1,53 & 1,26 & 1,03 & 1,71 \\
Glicina I & 0,91 & 0,64 & 0,71 & 0,4 & 0,25 & 0,87 & 0,63 & 0,78 \\
Alanina & 1,07 & 0,52 & 1,92 & 0,31 & 0,51 & 0,82 & 0,56 & 0,64 \\
Cistina & 0,25 & 0,25 & 0,23 & 0,14 & 0,19 & 0,2 & 0,15 & 0,23 \\
\hline 1: RR= ração-referência, TIM= trigo integral moído, GT= germe de trigo, FTC= farinha de trigo clara, FTE= farinha de trigo escura, \\
FTH= farelo de trigo para uso humano, FTA= farelo de trigo para uso animal, FTG= farelo de trigo grosso.
\end{tabular}

Da mesma forma que para os aminoácidos essenciais, as farinhas de trigo apresentaram os menores valores de aminoácidos não essenciais, com exceção à serina, glutamina e prolina. No trigo integral foram obtidos os mais altos valores de glutamina e de prolina.

Nas Tab. 3 e 4 encontram-se os valores dos coeficientes de digestibilidade aparente dos aminoácidos essenciais $\left(\mathrm{CDAaa}_{\mathrm{e}}\right)$ e não essenciais $\left(\mathrm{CDAaa}_{\mathrm{ne}}\right)$, respectivamente, segundo os tratamentos. Na

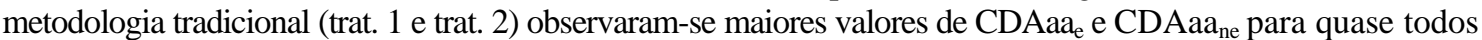
os aminoácidos, com exceção aos valores de valina dos alimentos FTH e FTG, que foram semelhantes ao tratamento 3 (Sibbald - aves não cecectomizadas), da isoleucina de RR e FTG, cujos valores foram iguais entre os tratamentos 1 (tradicional - pintos) e 3 (Sibbald - aves não cecectomizadas), de fenilalanina dos alimentos GT, FTE e FTG, cujos valores foram iguais entre os tratamentos 1 e 3, da histidina do GT, semelhante para todos os tratamentos, e da lisina do GT, semelhante para os tratamentos 1, 2 e 3 . Estes resultados foram semelhantes aos encontrados por Lee et al. (1995), que compararam as digestibilidades aparente e verdadeira dos aminoácidos do farelo de canola pela metodologia de alimentação à vontade (dieta basal) e alimentação forçada (alimento puro). Também ocorreram diferenças quanto ao CDAaae entre os tratamentos 1 e 2, com alguns resultados maiores no tratamento 1, como por exemplo valina no FTC, metionina na RR, isoleucina no FTH, leucina no TIM e FTH, treonina no FTH e lisina no RR e FTH. Ao contrário, alguns aminoácidos apresentaram maior coeficiente de digestibilidade no tratamento 2 em relação ao tratamento 1: metionina no FTA, isoleucina no FTG, fenilalanina na RR, FTE e FTG, histidina no TIM, FTC e FTE e lisina no TIM, FTC e FTE. 


\section{Borges et al.}

Tabela 3. Coeficiente de digestibilidade aparente (\%) dos aminoácidos essenciais dos alimentos ${ }^{1}$

\begin{tabular}{|c|c|c|c|c|c|c|c|c|c|}
\hline \multirow{2}{*}{ Aminoácido } & \multirow{2}{*}{ Método } & \multicolumn{8}{|c|}{ Alimento $^{1}$} \\
\hline & & RR & TIM & GT & FTC & FTE & FTH & FTA & FTG \\
\hline \multirow{4}{*}{ Valina } & Trat. 1 & $90,08 a$ & $93,29 a$ & $81,54 a$ & $91,09 a$ & $85,69 a$ & $78,37 a$ & $76,37 a$ & $69,52 a$ \\
\hline & Trat. 2 & $88,73 a$ & $89,50 a$ & $81,62 a$ & $83,38 b$ & $82,47 a$ & $74,02 a$ & $77,43 a$ & $75,46 a$ \\
\hline & Trat. 3 & $77,86 \mathrm{~b}$ & 74,73b & $65,44 \mathrm{~b}$ & 49,91c & $61,93 c$ & $71,46 a$ & $65,17 \mathrm{~b}$ & $75,25 a$ \\
\hline & Trat. 4 & $65,24 c$ & $48,93 c$ & $53,43 c$ & $42,19 d$ & $29,08 \mathrm{~d}$ & $51,71 b$ & $42,48 c$ & $45,92 b$ \\
\hline \multirow{4}{*}{ Metionina } & Trat. 1 & $97,21 a$ & $94,80 \mathrm{a}$ & $92,36 a$ & $94,03 a$ & $90,08 a$ & $93,55 a$ & $83,97 b$ & $88,04 a$ \\
\hline & Trat. 2 & $95,23 b$ & $91,39 a$ & $92,12 a$ & $91,77 a$ & $91,03 a$ & $88,45 a$ & $91,64 a$ & $89,13 a$ \\
\hline & Trat. 3 & $86,20 c$ & $73,60 \mathrm{~b}$ & $79,04 \mathrm{~b}$ & $54,19 \mathrm{~b}$ & $56,88 \mathrm{~b}$ & $71,87 b$ & $51,33 c$ & $62,65 b$ \\
\hline & Trat. 4 & $82,96 \mathrm{~d}$ & $61,60 \mathrm{c}$ & $69,53 c$ & $36,89 \mathrm{c}$ & $55,53 b$ & $56,73 c$ & $44,95 d$ & $59,74 b$ \\
\hline \multirow{4}{*}{ Isoleucina } & Trat. 1 & 84,88ab & $95,72 a$ & $89,57 a$ & $98,77 a$ & $87,14 a$ & $92,60 a$ & $81,29 a$ & $85,59 b$ \\
\hline & Trat. 2 & 87,82a & $93,46 a$ & $84,84 a b$ & $92,87 a$ & $86,80 a$ & $85,38 \mathrm{~b}$ & $77,67 a$ & $89,48 a$ \\
\hline & Trat. 3 & $80,54 b$ & 69,63b & $76,32 \mathrm{bc}$ & $82,37 b$ & $54,59 b$ & $84,07 b$ & $56,11 b$ & $85,23 b$ \\
\hline & Trat. 4 & 61,97c & $40,38 c$ & $70,37 \mathrm{c}$ & $67,25 c$ & $-18,46 c$ & $64,22 \mathrm{c}$ & $21,95 c$ & $65,51 \mathrm{c}$ \\
\hline \multirow{4}{*}{ Leucina } & Trat. 1 & $89,00 a$ & $94,58 a$ & $82,60 a$ & $88,20 a$ & $85,88 a$ & 81,70a & 75,78a & 69,17ab \\
\hline & Trat. 2 & $87,68 a$ & $87,26 b$ & $82,98 a$ & $85,80 a$ & $88,64 a$ & $68,93 \mathrm{~b}$ & $76,74 a$ & $76,38 a$ \\
\hline & Trat. 3 & $78,85 b$ & $59,05 c$ & 67,86b & $60,03 \mathrm{~b}$ & $60,37 b$ & $53,80 c$ & 37,52b & $56,69 b$ \\
\hline & Trat. 4 & $61,38 \mathrm{c}$ & $37,51 d$ & $59,28 c$ & $20,77 \mathrm{c}$ & $8,95 c$ & $6,80 \mathrm{~d}$ & $9,74 \mathrm{c}$ & $9,06 \mathrm{c}$ \\
\hline \multirow{4}{*}{ Treonina } & Trat. 1 & $93,12 a$ & $90,76 a$ & $87,47 a$ & $88,57 a$ & $88,38 a$ & $82,54 a$ & $79,07 a$ & $79,58 a$ \\
\hline & Trat. 2 & $92,32 a$ & $90,51 \mathrm{a}$ & $84,29 a$ & $81,95 b$ & $85,63 a$ & $75,12 b$ & $80,33 a$ & $80,76 a$ \\
\hline & Trat. 3 & $72,41 b$ & $44,89 b$ & $68,03 \mathrm{~b}$ & $11,25 c$ & $36,42 b$ & $49,85 c$ & $30,77 b$ & $54,12 b$ \\
\hline & Trat. 4 & $52,09 c$ & $5,65 c$ & $50,91 c$ & $-70,58 d$ & $-13,48 c$ & $3,185 d$ & $0,465 c$ & $12,52 \mathrm{c}$ \\
\hline \multirow{4}{*}{ Fenilalanina } & Trat. 1 & $82,92 b$ & $82,15 a$ & 69,73a & 75,39a & $62,58 b$ & $62,07 a$ & $66,68 a$ & $56,64 b$ \\
\hline & Trat. 2 & $86,44 a$ & $86,63 a$ & $71,15 a$ & $79,45 a$ & $79,31 \mathrm{a}$ & $63,64 a$ & $60,50 a$ & $68,01 \mathrm{a}$ \\
\hline & Trat. 3 & 73,97c & $58,66 \mathrm{~b}$ & $64,20 \mathrm{a}$ & $61,21 b$ & $61,17 b$ & $48,44 b$ & $36,49 b$ & $54,26 \mathrm{~b}$ \\
\hline & Trat. 4 & $55,77 d$ & $40,29 c$ & $53,74 a$ & $30,42 \mathrm{c}$ & $23,17 \mathrm{c}$ & $29,79 c$ & $22,37 \mathrm{c}$ & $35,71 c$ \\
\hline \multirow{4}{*}{ Histidina } & Trat. 1 & $92,04 a$ & $87,98 b$ & $88,20 a$ & $85,66 b$ & $86,24 b$ & 79,69a & $72,92 a$ & 71,99ab \\
\hline & Trat. 2 & $91,93 a$ & $95,51 \mathrm{a}$ & $92,93 a$ & $92,28 a$ & $94,48 a$ & $82,68 a$ & $83,28 a$ & $81,32 a$ \\
\hline & Trat. 3 & $69,26 \mathrm{~b}$ & $60,59 c$ & 77,79a & $49,33 c$ & $55,09 c$ & $56,29 b$ & 41,14b & 61,99b \\
\hline & Trat. 4 & $52,88 \mathrm{c}$ & $35,81 d$ & $75,31 a$ & $8,24 d$ & $27,88 \mathrm{~d}$ & $14,02 \mathrm{c}$ & $26,55 c$ & $29,02 c$ \\
\hline \multirow{4}{*}{ Lisina } & Trat. 1 & $93,40 a$ & $87,29 b$ & $90,54 a$ & $87,65 b$ & $86,19 b$ & $89,47 a$ & 79,36a & $79,05 a$ \\
\hline & Trat. 2 & $90,90 \mathrm{~b}$ & $94,54 a$ & 89,90a & $94,43 a$ & $94,47 a$ & $82,32 b$ & $84,92 a$ & $89,42 a$ \\
\hline & Trat. 3 & $82,03 c$ & $58,97 \mathrm{c}$ & 86,96ab & $48,45 c$ & $57,85 \mathrm{c}$ & $63,40 c$ & $52,69 b$ & $55,50 \mathrm{~b}$ \\
\hline & Trat. 4 & $79,27 d$ & $41,54 d$ & $85,77 \mathrm{~b}$ & $11,04 d$ & $43,85 d$ & $60,55 c$ & $47,08 \mathrm{~b}$ & $51,59 \mathrm{~b}$ \\
\hline
\end{tabular}

Valores na mesma coluna, para uma mesma variável, seguidos de letras distintas diferem entre si pelo teste SNK $(\mathrm{P}<0,05)$

1: RR= ração-referência, TIM= trigo integral moído, GT= germe de trigo, FTC= farinha de trigo clara, FTE= farinha de trigo escura,

$\mathrm{FTH}=$ farelo de trigo para uso humano, FTA= farelo de trigo para uso animal, FTG= farelo de trigo grosso. 
Avaliação dos coeficientes de digestibilidade...

Tabela 4. Coeficiente de digestibilidade aparente (\%) dos aminoácidos não essenciais dos alimentos

\begin{tabular}{|c|c|c|c|c|c|c|c|c|c|}
\hline \multirow{2}{*}{ Aminoácido } & \multirow{2}{*}{ Método } & \multicolumn{8}{|c|}{ Alimento $^{1}$} \\
\hline & & RR & TIM & GT & FTC & FTE & FTH & FTA & FTG \\
\hline \multirow{4}{*}{ Aspartato } & Trat. 1 & $92,20 a$ & $82,04 a$ & $89,67 a$ & $86,16 a$ & $86,93 a$ & $84,84 a$ & $76,50 a$ & $70,61 b$ \\
\hline & Trat. 2 & $89,43 b$ & $85,15 a$ & $89,01 \mathrm{a}$ & $89,73 a$ & $83,08 a$ & $79,01 b$ & $74,94 a$ & $80,68 a$ \\
\hline & Trat. 3 & $82,05 c$ & 42,63b & $77,38 b$ & $12,01 \mathrm{~b}$ & $33,87 b$ & 50,91c & 34,26b & $32,43 c$ \\
\hline & Trat. 4 & $67,43 d$ & $16,25 c$ & $75,00 \mathrm{~b}$ & $-29,29 c$ & $15,99 \mathrm{c}$ & $28,25 d$ & $25,72 b$ & $31,41 \mathrm{c}$ \\
\hline \multirow{4}{*}{ Tirosina } & Trat. 1 & 87,40a & $84,64 a$ & $81,80 a$ & $84,79 a$ & $81,49 a$ & $76,05 a$ & 71,82a & $62,25 a$ \\
\hline & Trat. 2 & $86,27 a$ & $87,52 a$ & $83,23 a$ & $86,47 a$ & $85,44 a$ & $74,30 \mathrm{a}$ & $74,22 a$ & $77,48 a$ \\
\hline & Trat. 3 & $73,76 b$ & $48,03 b$ & $69,55 b$ & $30,41 b$ & $39,78 b$ & $52,72 b$ & $37,84 \mathrm{~b}$ & $59,49 b$ \\
\hline & Trat. 4 & $55,14 \mathrm{c}$ & $19,16 \mathrm{c}$ & $64,51 b$ & $0,55 c$ & $3,14 \mathrm{c}$ & $24,85 c$ & $22,72 \mathrm{c}$ & $21,65 c$ \\
\hline \multirow{4}{*}{ Serina } & Trat. 1 & $89,67 a$ & $89,79 a$ & $86,55 a$ & 89,64a & $88,69 a$ & $80,89 a$ & $77,25 a$ & $70,44 \mathrm{~b}$ \\
\hline & Trat. 2 & $88,61 \mathrm{a}$ & $93,03 a$ & $86,56 a$ & $89,59 a$ & $90,07 a$ & $73,91 b$ & $76,96 a$ & $77,74 a$ \\
\hline & Trat. 3 & $75,60 \mathrm{~b}$ & $55,72 b$ & $71,36 b$ & $61,76 b$ & $65,05 b$ & $47,80 \mathrm{c}$ & $36,79 b$ & $54,41 \mathrm{c}$ \\
\hline & Trat. 4 & $63,83 c$ & $46,19 c$ & $70,21 b$ & $37,50 \mathrm{c}$ & $44,96 \mathrm{c}$ & $37,74 d$ & $35,60 \mathrm{~b}$ & $42,37 d$ \\
\hline \multirow{4}{*}{ Glicina } & Trat. 1 & $94,21 \mathrm{a}$ & $98,75 a$ & $93,96 a$ & $97,28 \mathrm{a}$ & $96,20 a$ & $91,03 a$ & $87,34 a$ & $86,12 a$ \\
\hline & Trat. 2 & $93,06 a$ & $99,67 a$ & $92,51 \mathrm{a}$ & $94,72 b$ & $95,73 a$ & $81,29 b$ & $86,70 a$ & $87,70 \mathrm{a}$ \\
\hline & Trat. 3 & $86,80 \mathrm{~b}$ & $89,11 b$ & $83,93 b$ & $91,14 \mathrm{c}$ & $90,66 b$ & $76,83 c$ & $73,68 b$ & $86,09 a$ \\
\hline & Trat. 4 & $78,19 c$ & $85,44 c$ & $83,48 b$ & $84,14 d$ & 84,65 & $71,17 d$ & $69,78 c$ & $77,79 b$ \\
\hline \multirow{4}{*}{ Prolina } & Trat. 1 & $93,03 a$ & $93,48 b$ & $85,72 a$ & $91,63 b$ & $90,45 a$ & $83,49 b$ & $82,92 a$ & $77,54 \mathrm{~b}$ \\
\hline & Trat. 2 & $91,03 a$ & $98,77 a$ & $87,02 a$ & $100,14 a$ & $98,19 b$ & $92,92 a$ & $86,83 a$ & $97,83 a$ \\
\hline & Trat. 3 & 79,00b & $78,88 \mathrm{c}$ & $67,13 b$ & $74,89 c$ & $72,58 \mathrm{c}$ & $61,21 \mathrm{c}$ & $61,16 b$ & $77,25 b$ \\
\hline & Trat. 4 & $63,99 c$ & $71,09 d$ & $65,34 b$ & $70,02 c$ & $62,11 d$ & $46,26 \mathrm{~d}$ & $47,10 \mathrm{c}$ & $67,23 c$ \\
\hline \multirow{4}{*}{ Alanina } & Trat. 1 & 83,93a & $88,58 a$ & $95,78 a$ & $89,22 a$ & $86,47 a$ & 74,89a & 68,82a & $54,27 a$ \\
\hline & Trat. 2 & $82,98 a$ & $85,94 a$ & $90,84 a$ & $61,87 b$ & $75,88 a$ & $42,89 b$ & $49,06 \mathrm{~b}$ & $32,70 \mathrm{~b}$ \\
\hline & Trat. 3 & $69,73 b$ & $38,25 b$ & $78,56 b$ & $-1,82 c$ & $45,42 b$ & $38,67 b$ & $24,03 c$ & $41,70 \mathrm{~b}$ \\
\hline & Trat. 4 & $60,03 c$ & $14,78 \mathrm{c}$ & $77,38 b$ & $-2,39 c$ & $33,32 b$ & $36,82 b$ & $19,58 \mathrm{c}$ & $15,28 \mathrm{c}$ \\
\hline
\end{tabular}

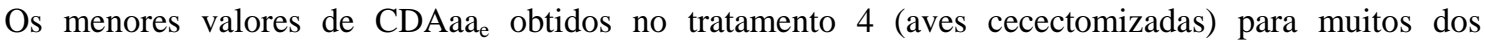
aminoácidos, inclusive com resultados negativos, possivelmente são atribuídos à influência das perdas metabólica e endógena, aliada ao baixo consumo de alimentos. Além disso, as aves do tratamento 4 eram mais pesadas, justificando a maior perda endógena e metabólica, o que justificaria as diferenças encontradas entre os tratamentos 3 e 4. Segundo Johns et al. (1986a), os baixos coeficientes de CDAaa obtidos em aves adultas são resultados do aumento do nitrogênio endógeno com o aumento de peso e idade. O nitrogênio endógeno inclui enzimas digestivas, glicoproteínas (mucina), células de descamação, aminoácidos produzidos pelo catabolismo celular e albuminas séricas. Como ocorre aumento do nitrogênio na excreta, os valores de digestibilidade aparente conseqüentemente diminuem com a idade. Albino (1991) não encontrou resultados negativos quando avaliou o CDAaa para pintos e galos, utilizando metodologias tradicional e de alimentação forçada, respectivamente, embora tenha obtido valores menores de CDAaa de vários aminoácidos para galos, quando usou a metodologia de alimentação forçada.

Na Tab. 5 encontram-se os valores de digestibilidade aparente da soma total dos aminoácidos, da soma dos aminoácidos não essenciais, da soma dos aminoácidos essenciais e da soma dos aminoácidos limitantes (MET + TRE + LIS), segundo os tratamentos. Na metodologia tradicional observaram-se maiores valores de CDAaa em todos os alimentos quando comparados aos valores obtidos no método de alimentação forçada, novamente atribuídos à interferência das perdas metabólicas e do baixo consumo nos métodos de alimentação forçada. Em alguns alimentos esses valores foram negativos, mostrando que a perda endógena foi maior do que a ingestão de aminoácidos. Os alimentos altos em fibra (FTH, FTA e 


\section{Borges et al.}

FTG) mostraram tendência a apresentar menores valores de CDAaa tanto na metodologia tradicional como na de alimentação forçada, confirmando a interferência da fibra na digestibilidade dos aminoácidos.

Tabela 5. Coeficiente de digestibilidade aparente (\%) da soma total dos aminoácidos (AA), da soma dos AA não essenciais, da soma dos AA essenciais e da soma dos AA limitantes dos alimentos

\begin{tabular}{|c|c|c|c|c|c|c|c|c|c|}
\hline \multirow{2}{*}{ Aminoácido } & \multirow{2}{*}{ Método } & \multicolumn{8}{|c|}{ Alimento $^{1}$} \\
\hline & & RR & TIM & GT & FTC & FTE & FTH & FTA & FTG \\
\hline \multirow{4}{*}{ AA totais } & Trat. 1 & $90,14 a$ & $89,75 a$ & $86,82 a$ & $89,15 a$ & $85,69 a$ & $81,72 a$ & $77,15 a$ & $72,91 b$ \\
\hline & Trat. 2 & $89,53 a$ & $91,87 a$ & $86,36 a$ & $87,46 a$ & $88,14 a$ & $75,87 b$ & $77,00 \mathrm{a}$ & $78,87 a$ \\
\hline & Trat. 3 & $77,72 b$ & $60,91 b$ & $72,01 b$ & $48,95 b$ & $56,45 b$ & $59,64 c$ & $44,39 b$ & $61,02 \mathrm{c}$ \\
\hline & Trat. 4 & $64,23 c$ & $40,33 c$ & $69,98 b$ & $21,91 \mathrm{c}$ & $28,72 \mathrm{c}$ & $38,14 d$ & $32,41 c$ & $40,55 d$ \\
\hline \multirow{4}{*}{$\mathrm{AA}_{\mathrm{ne}}$} & Trat. 1 & $89,91 a$ & $89,54 a$ & 88,91a & $89,79 a$ & $88,38 a$ & $81,87 a$ & $77,49 a$ & $70,20 \mathrm{~b}$ \\
\hline & Trat. 2 & $88,72 a$ & $91,68 a$ & $88,20 a$ & $87,09 a$ & $88,06 a$ & $75,05 b$ & $74,24 a$ & $75,79 a$ \\
\hline & Trat. 3 & $77,82 b$ & $58,77 \mathrm{~b}$ & $74,19 b$ & $44,73 b$ & $57,89 \mathrm{~b}$ & $55,33 c$ & $43,69 b$ & $58,58 \mathrm{c}$ \\
\hline & Trat. 4 & $64,76 \mathrm{c}$ & $42,16 c$ & $73,12 b$ & $26,75 c$ & $40,70 \mathrm{c}$ & $41,15 d$ & $37,69 c$ & $42,62 d$ \\
\hline \multirow{4}{*}{$\mathrm{AA}_{\mathrm{e}}$} & Trat. 1 & $90,32 a$ & $89,90 a$ & $85,25 a$ & $88,67 a$ & $83,68 a$ & $81,61 a$ & 76,93a & $74,94 b$ \\
\hline & Trat. 2 & $90,13 a$ & $92,02 a$ & $84,97 a$ & $87,74 a$ & $88,20 a$ & $77,98 b$ & $79,06 a$ & $81,25 a$ \\
\hline & Trat. 3 & $77,64 b$ & $62,52 b$ & $70,37 b$ & $52,10 \mathrm{~b}$ & $55,37 b$ & $62,88 \mathrm{c}$ & $44,91 b$ & $62,85 c$ \\
\hline & Trat. 4 & $63,94 \mathrm{c}$ & $38,96 \mathrm{c}$ & $67,63 b$ & $18,28 \mathrm{c}$ & $19,73 c$ & $35,88 d$ & $28,44 c$ & $39,00 d$ \\
\hline \multirow{4}{*}{$\mathrm{LIM}^{2}$} & Trat. 1 & $94,58 a$ & $90,94 a$ & $90,12 a$ & $90,09 a$ & $87,30 \mathrm{~b}$ & $86,14 a$ & $80,80 a$ & $82,22 a$ \\
\hline & Trat. 2 & $92,82 a$ & $92,15 a$ & $88,77 a$ & $89,39 a$ & $91,30 \mathrm{a}$ & $84,35 a$ & $85,83 a$ & $86,44 a$ \\
\hline & Trat. 3 & $80,2 b$ & $59,15 b$ & $74,44 b$ & $37,97 b$ & $49,93 c$ & $61,71 b$ & 40,93b & $56,45 b$ \\
\hline & Trat. 4 & $71,44 \mathrm{c}$ & $36,26 c$ & $72,39 b$ & $-7,55 c$ & $29,08 \mathrm{~d}$ & $40,16 \mathrm{c}$ & $34,86 c$ & $42,26 c$ \\
\hline
\end{tabular}

Valores na mesma coluna, para uma mesma variável, seguidos de letras distintas diferem entre si pelo teste $\mathrm{SNK}(\mathrm{P}<0,05)$

1: RR= ração-referência, TIM= trigo integral moído, GT= germe de trigo, FTC= farinha de trigo clara, FTE= farinha de trigo escura, $\mathrm{FTH}=$ farelo de trigo para uso humano, FTA= farelo de trigo para uso animal, FTG= farelo de trigo grosso.

2: Metionina, treonina e lisina.

Os alimentos com alto teor de fibra (FTH, FTA e FTG) apresentaram, para a maioria dos aminoácidos, os menores valores absolutos de $\mathrm{CDVaa}_{\mathrm{e}}$ (Tab. 6). Entre os tratamentos com metodologia tradicional poucas diferenças foram significativas (oito aminoácidos apresentaram maiores CDAaa para T1 e oito valores mais altos de digestibilidade verdadeira para T2). As aves não cecectomizadas (tratamento 3) apresentaram, em sua grande maioria, valores iguais ou menores do que aqueles dos tratamentos de metodologia tradicional. Albino (1991) encontrou, para a maioria dos aminoácidos, valores maiores de digestibilidade verdadeira para galos do que para pintos e sugeriu que essas diferenças podiam ser atribuídas às metodologias utilizadas.

No método tradicional com pintos a isoleucina do tratamento FTC o valor de CDVaa foi superior a 100\%. Likuski e Dorell (1978) verificaram valores acima de 100\% para triptofano, cistina, metionina, lisina, tirosina, histidina e serina da soja e da cevada. A explicação poderia ser as pequenas concentrações desses aminoácidos nos alimentos e na excreta. Da mesma forma, Bragg et al. (1970) encontraram digestibilidade maior do que $100 \%$ para cistina, ao trabalharem com aves modificadas cirurgicamente, sugerindo que elas podem secretar maior quantidade de nitrogênio no intestino. Neste trabalho, as aves cecectomizadas (tratamento 4) apresentaram, de modo geral, CDVaa inferiores aos das aves não cecectomizadas, o que pode ser atribuído à reduzida fermentação microbiana. Estes resultados sugerem substancial desaminação de aminoácidos a amônia no ceco das aves não cecectomizadas e não concordam com os resultados obtidos por Han e Parsons (1990) e Johns et al. (1986b), que encontraram maiores valores de aminoácidos digestíveis em aves cecectomizadas em relação aos de aves não cecectomizadas. 
Avaliação dos coeficientes de digestibilidade...

Tabela 6. Coeficientes de digestibilidade verdadeira (\%) dos aminoácidos essenciais $\left(\mathrm{CDVaa}_{\mathrm{e}}\right)$ dos alimentos

\begin{tabular}{|c|c|c|c|c|c|c|c|c|c|}
\hline \multirow{2}{*}{ Aminoácido } & \multirow{2}{*}{ Método } & \multicolumn{8}{|c|}{ Alimento $^{1}$} \\
\hline & & $\mathrm{RR}$ & TIM & GT & FTC & FTE & FTH & FTA & FTG \\
\hline \multirow{4}{*}{ Valina } & Trat. 1 & $91,21 a$ & $94,64 a$ & $83,31 a$ & $93,18 a$ & $87,85 b$ & $80,19 b$ & $78,30 \mathrm{~b}$ & $71,46 b$ \\
\hline & Trat. 2 & $89,36 a$ & $90,18 a$ & $82,17 a$ & $84,72 b$ & $83,56 b$ & $72,28 b$ & $78,17 \mathrm{~b}$ & $76,22 b$ \\
\hline & Trat. 3 & $90,73 a$ & $94,43 a$ & $68,23 b$ & $80,35 c$ & $97,62 a$ & $90,84 a$ & $87,98 a$ & $94,52 a$ \\
\hline & Trat. 4 & $80,28 b$ & $71,76 b$ & $82,73 a$ & $77,76 \mathrm{~d}$ & $70,79 c$ & $71,37 \mathrm{c}$ & $69,14 \mathrm{~b}$ & $68,44 c$ \\
\hline \multirow{4}{*}{ Metionina } & Trat. 1 & $98,12 \mathrm{a}$ & $96,05 a$ & $93,83 a$ & $95,82 \mathrm{a}$ & $91,78 a$ & $95,29 a$ & $85,73 b$ & $89,82 a$ \\
\hline & Trat. 2 & $95,61 b$ & $91,88 \mathrm{~b}$ & $92,49 a$ & $92,66 a$ & $91,71 \mathrm{a}$ & $89,07 a b$ & $92,18 a$ & $89,69 a$ \\
\hline & Trat. 3 & $97,17 a$ & $97,42 \mathrm{a}$ & $84,08 \mathrm{~b}$ & $92,07 a$ & $90,52 a$ & $94,09 a$ & $75,69 d$ & $85,32 b$ \\
\hline & Trat. 4 & $94,06 c$ & $85,69 c$ & $93,76 a$ & $75,22 b$ & $92,28 a$ & $79,22 b$ & $82,43 c$ & $88,54 a$ \\
\hline \multirow{4}{*}{ Isoleucina } & Trat. 1 & $86,32 a$ & $97,49 a$ & $91,28 a$ & $100,44 a$ & $89,96 a$ & $94,07 a$ & $83,87 a$ & 87,09ab \\
\hline & Trat. 2 & $88,99 a$ & $94,84 a$ & $85,58 a$ & $94,58 \mathrm{ab}$ & $88,99 a$ & $85,04 \mathrm{c}$ & $79,23 a$ & $90,33 a$ \\
\hline & Trat. 3 & $86,00 a$ & $79,29 b$ & $81,19 a$ & $88,88 \mathrm{~b}$ & $74,83 \mathrm{~b}$ & $89,91 b$ & $68,36 \mathrm{~b}$ & $90,09 a$ \\
\hline & Trat. 4 & $83,51 a$ & $78,50 \mathrm{~b}$ & $89,56 a$ & $92,96 a b$ & $61,41 \mathrm{c}$ & $82,09 d$ & $70,29 b$ & $84,68 b$ \\
\hline \multirow{4}{*}{ Leucina } & Trat. 1 & $89,89 a$ & 88,37ab & 83,92ab & $89,81 a$ & $87,40 \mathrm{a}$ & $83,34 a$ & $77,41 a$ & 70,78a \\
\hline & Trat. 2 & $88,18 a$ & $95,18 a$ & $83,35 a b$ & $86,90 a$ & $89,45 a$ & $69,73 \mathrm{~b}$ & $77,42 a$ & $77,08 a$ \\
\hline & Trat. 3 & $86,48 a$ & $72,75 c$ & $76,65 b$ & $79,79 a$ & $78,91 a$ & $67,88 \mathrm{~b}$ & $55,42 a$ & 71,33a \\
\hline & Trat. 4 & $88,32 a$ & $85,84 b$ & $90,28 a$ & $90,48 a$ & $74,38 \mathrm{a}$ & $56,48 \mathrm{c}$ & $72,87 a$ & $60,73 a$ \\
\hline \multirow{4}{*}{ Treonina } & Trat. 1 & $94,49 a$ & $92,61 \mathrm{a}$ & $89,36 a$ & 91,39a & $87,98 a$ & $85,02 a$ & $81,54 a$ & $81,98 a$ \\
\hline & Trat. 2 & $92,96 a$ & $91,33 a$ & $84,79 a$ & $83,52 b$ & $89,43 a$ & $76,10 \mathrm{~b}$ & $81,18 a$ & $81,61 a$ \\
\hline & Trat. 3 & $93,06 a$ & $87,52 \mathrm{a}$ & $89,85 a$ & $93,96 a$ & $87,96 a$ & $86,50 \mathrm{a}$ & $78,25 a$ & $91,26 a$ \\
\hline & Trat. 4 & $85,74 b$ & $75,11 b$ & $86,45 a$ & $64,16 \mathrm{c}$ & $70,51 b$ & $62,89 \mathrm{c}$ & $77,82 a$ & 73,03a \\
\hline \multirow{4}{*}{ Fenilalanina } & Trat. 1 & $84,39 a$ & $83,72 a$ & 71,74a & $77,82 \mathrm{a}$ & 64,89c & $64,59 a$ & $69,14 a$ & $59,11 \mathrm{c}$ \\
\hline & Trat. 2 & $87,07 a$ & $87,27 a$ & $71,63 a$ & $80,71 a$ & $80,24 a b$ & $64,54 a$ & $61,25 a$ & $68,80 \mathrm{~b}$ \\
\hline & Trat. 3 & $85,31 a$ & $75,06 \mathrm{~b}$ & $76,01 \mathrm{a}$ & $85,12 a$ & $84,25 a$ & $66,57 a$ & $58,25 a$ & 73,14ab \\
\hline & Trat. 4 & $80,59 b$ & $76,20 \mathrm{~b}$ & $79,59 a$ & $82,64 a$ & $73,71 \mathrm{~b}$ & $69,48 a$ & $70,01 \mathrm{a}$ & $77,01 \mathrm{a}$ \\
\hline \multirow{4}{*}{ Histidina } & Trat. 1 & $94,50 a$ & $90,83 a$ & $90,51 a$ & $90,10 \mathrm{c}$ & $90,20 \mathrm{~b}$ & $83,48 a$ & 77,74ab & 75,90ab \\
\hline & Trat. 2 & $92,56 a$ & $96,21 a$ & $93,20 \mathrm{a}$ & $93,64 b$ & $95,44 a$ & $83,50 a$ & $83,98 a$ & $82,07 a b$ \\
\hline & Trat. 3 & $88,30 \mathrm{~b}$ & $91,00 a$ & $91,44 a$ & $98,40 a$ & $95,43 a$ & $81,80 a$ & 72,84ab & $90,55 a$ \\
\hline & Trat. 4 & $76,65 c$ & $73,77 b$ & $92,36 a$ & $69,52 d$ & $68,26 \mathrm{c}$ & $45,86 b$ & $66,13 b$ & $64,67 b$ \\
\hline \multirow{4}{*}{ Lisina } & Trat. 1 & $94,42 a$ & $88,85 b$ & $91,46 b$ & $89,84 b$ & $88,18 b$ & $84,08 \mathrm{~b}$ & $81,16 a$ & $81,03 a$ \\
\hline & Trat. 2 & $91,37 b$ & $95,24 a$ & $90,09 b$ & $95,64 a$ & $95,34 a$ & $90,16 a$ & $85,54 a$ & $90,13 a$ \\
\hline & Trat. 3 & $91,57 b$ & $87,13 b$ & $92,44 b$ & $97,36 a$ & $92,30 \mathrm{ab}$ & $78,94 \mathrm{~b}$ & $67,82 \mathrm{~b}$ & $77,24 a$ \\
\hline & Trat. 4 & $92,75 b$ & $81,28 \mathrm{c}$ & $96,36 a$ & $80,05 c$ & $92,48 a b$ & $82,48 b$ & $81,95 a$ & $82,27 a$ \\
\hline
\end{tabular}

Segundo Johns et al. (1986a), a flora microbiana modifica a composição da excreta por degradar os aminoácidos da digesta e sintetizar outros produtos nitrogenados, entretanto a taxa de degradação microbiana é maior que a taxa de síntese. Desse modo, a microflora do ceco pode ser responsável pela degradação de parte dos aminoácidos que chegam a ele, superestimando os valores de CDVaa ${ }_{e}$ nas aves não cecectomizadas.

Em geral, os tratamentos com metodologia tradicional não apresentaram diferenças estatísticas entre si quanto ao CDVaa ; dos 64 pares de coeficientes, só 17 apresentaram diferenças $(\mathrm{P}<0,05)$. Entre os tratamentos de alimentação forçada, 38 pares apresentaram diferenças significativas.

Quanto ao $\mathrm{CDVaa}_{\text {ne }}$, dos 48 coeficientes de alimentação tradicional, 33 foram semelhantes entre si e 15 apresentaram diferenças significativas (Tab. 7). Nos tratamentos de alimentação forçada, 19 foram semelhantes entre si e 29 apresentaram diferenças estatísticas. Para alguns aminoácidos, como aspartato, 


\section{Borges et al.}

tirosina, prolina e alanina, os valores de $\mathrm{CDVaa}_{\mathrm{ne}}$ das aves cecectomizadas foram menores dos que os de aves não cecectomizadas. Quando se compararam os tratamentos de metodologia tradicional aos de alimentação forçada observa-se que os maiores coeficientes de digestibilidade dos aminoácidos foram obtidos nos primeiros, embora as aves normais que receberam alimentação forçada apresentassem alguns valores de aminoácidos maiores. Os farelos de trigo apresentaram os menores valores de CDVaa ne $_{\text {, }}$ sobretudo para alanina, e isso provavelmente foi devido aos altos teores de fibra contidos nesses alimentos.

Tabela 7. Coeficiente de digestibilidade verdadeira (\%) dos aminoácidos não essenciais (CDVaa ${ }_{\text {ne }}$ dos alimentos

\begin{tabular}{|c|c|c|c|c|c|c|c|c|c|}
\hline \multirow{2}{*}{ Aminoácido } & \multirow{2}{*}{ Método } & \multicolumn{8}{|c|}{ Alimento $^{1}$} \\
\hline & & RR & TIM & GT & FTC & FTE & FTH & FTA & FTG \\
\hline \multirow{4}{*}{ Aspartato } & Trat. 1 & $92,96 a$ & $83,28 a$ & $90,56 a b$ & $87,55 a$ & $8843 a$ & $86,31 a$ & $77,96 a$ & $72,16 b$ \\
\hline & Trat. 2 & $89,88 \mathrm{ab}$ & $85,86 a$ & $89,28 a b$ & $90,92 a$ & $83,92 a$ & $79,75 b$ & $71,78 a$ & $81,39 a$ \\
\hline & Trat. 3 & $91,53 a$ & $73,30 \mathrm{~b}$ & $85,60 \mathrm{~b}$ & $64,69 c$ & $68,72 b$ & $69,94 \mathrm{~b}$ & $59,24 b$ & $56,26 \mathrm{c}$ \\
\hline & Trat. 4 & $87,40 \mathrm{~b}$ & $80,90 a$ & $92,31 \mathrm{a}$ & $81,73 b$ & $89,45 a$ & $68,35 b c$ & $78,35 a$ & $81,64 a$ \\
\hline \multirow{4}{*}{ Tirosina } & Trat. 1 & $82,52 b$ & $86,07 a$ & $83,05 a$ & $86,90 a$ & $83,44 b$ & $77,90 \mathrm{~b}$ & 73,70a & $64,19 \mathrm{c}$ \\
\hline & Trat. 2 & $86,95 b$ & $88,35 a$ & $83,63 a$ & $88,01 a$ & $86,56 b$ & $75,26 b$ & $75,76 a$ & $78,38 b$ \\
\hline & Trat. 3 & $94,28 a$ & $85,73 a$ & $86,60 \mathrm{a}$ & $89,40 a$ & $91,48 a$ & $83,21 a$ & $77,12 a$ & $95,15 a$ \\
\hline & Trat. 4 & $81,59 \mathrm{c}$ & $67,76 b$ & $86,50 a$ & $76,60 \mathrm{~b}$ & $69,79 c$ & $64,14 \mathrm{c}$ & $73,35 a$ & $67,62 \mathrm{c}$ \\
\hline \multirow{4}{*}{ Serina } & Trat. 1 & $90,84 a$ & $91,09 a$ & $87,90 a$ & $91,60 \mathrm{a}$ & $90,41 \mathrm{a}$ & $82,85 a$ & $79,23 a$ & $72,36 b$ \\
\hline & Trat. 2 & $89,31 \mathrm{ab}$ & $93,75 a$ & $86,97 a$ & $90,98 a$ & $91,03 a$ & $74,90 \mathrm{~b}$ & $77,82 a$ & $78,60 \mathrm{a}$ \\
\hline & Trat. 3 & $89,88 \mathrm{ab}$ & $77,07 \mathrm{~b}$ & $82,45 a$ & $92,59 a$ & $90,35 a$ & $69,71 \mathrm{c}$ & $63,50 \mathrm{~b}$ & $76,72 \mathrm{ab}$ \\
\hline & Trat. 4 & $85,59 b$ & $78,69 b$ & $90,00 \mathrm{a}$ & $84,45 a$ & $83,49 b$ & $71,10 \mathrm{bc}$ & $79,28 a$ & $76,65 \mathrm{ab}$ \\
\hline \multirow{4}{*}{ Glutamina } & Trat. 1 & $92,82 a$ & $99,04 a$ & $94,73 a$ & $87,88 a$ & $96,76 a$ & $91,96 a$ & $88,26 a$ & $86,81 b$ \\
\hline & Trat. 2 & $93,40 a$ & $99,81 a$ & $92,75 a$ & $95,16 \mathrm{~b}$ & $96,03 a$ & $81,74 b$ & $87,07 a$ & $87,99 b$ \\
\hline & Trat. 3 & $92,93 a$ & $93,65 b$ & $89,21 a$ & $97,10 \mathrm{ab}$ & $96,60 \mathrm{a}$ & $84,88 b$ & $83,13 b$ & $92,19 a$ \\
\hline & Trat. 4 & $88,95 b$ & $93,42 b$ & $94,00 \mathrm{a}$ & $94,62 b$ & $95,08 a$ & $85,32 b$ & $86,39 a$ & $88,47 b$ \\
\hline \multirow{4}{*}{ Prolina } & Trat. 1 & $93,92 b$ & $93,93 b$ & $86,90 a$ & $92,50 \mathrm{c}$ & $91,37 b$ & $84,82 \mathrm{c}$ & $84,27 b$ & $78,58 \mathrm{c}$ \\
\hline & Trat. 2 & $91,55 b$ & $99,01 a$ & $87,40 \mathrm{a}$ & $99,79 a$ & $98,68 a$ & $93,58 a$ & 87,39ab & $98,25 a$ \\
\hline & Trat. 3 & $99,79 a$ & $95,18 b$ & $89,09 a$ & $96,42 b$ & $96,14 a$ & $89,43 b$ & 95,63a & $98,44 a$ \\
\hline & Trat. 4 & $85,67 \mathrm{c}$ & $87,31 \mathrm{c}$ & $87,21 a$ & $91,47 \mathrm{c}$ & $85,59 c$ & $74,36 \mathrm{~d}$ & $81,42 b$ & $88,33 b$ \\
\hline \multirow{4}{*}{ Alanina } & Trat. 1 & $84,36 b$ & $90,45 a$ & $96,66 a$ & $91,96 a$ & $88,68 a$ & $76,97 a$ & $71,11 \mathrm{a}$ & $56,61 b$ \\
\hline & Trat. 2 & $84,66 b$ & $86,88 a$ & $90,99 a$ & $63,60 \mathrm{~d}$ & $77,00 \mathrm{a}$ & $37,75 b$ & $49,94 a$ & $33,64 c$ \\
\hline & Trat. 3 & $92,03 a$ & $84,76 a$ & $89,73 a$ & $75,74 b$ & $92,56 a$ & $71,46 a$ & $61,62 a$ & 79,33a \\
\hline & Trat. 4 & $80,33 b$ & $57,12 b$ & $89,81 a$ & $68,21 \mathrm{c}$ & $76,26 a$ & $65,07 a$ & $62,30 \mathrm{a}$ & $49,28 b$ \\
\hline
\end{tabular}

Valores na mesma coluna, para uma mesma variável, seguidos de letras distintas diferem entre si pelo teste $\mathrm{SNK}(\mathrm{P}<0,05)$

1: RR= ração-referência, TIM= trigo integral moído, GT= germe de trigo, FTC= farinha de trigo clara, FTE= farinha de trigo escura, $\mathrm{FTH}=$ farelo de trigo para uso humano, FTA= farelo de trigo para uso animal, FTG= farelo de trigo grosso.

Na Tab. 8 encontram-se os valores de digestibilidade verdadeira (CDVaa) da soma total dos aminoácidos, da soma dos aminoácidos não essenciais, dos aminoácidos essenciais, e da soma dos aminoácidos limitantes (MET + TRE + LIS), segundo os tratamentos. Do mesmo modo que em CDAaa, os alimentos altos em fibra (FTH, FTA e FTG) apresentaram os menores valores absolutos CDVaa em quase todos os tratamentos, confirmando a interferência da fibra sobre a digestibilidade dos aminoácidos. As diferenças foram menores quando se compararam os métodos tradicional e de alimentação forçada. As aves cecectomizadas apresentaram menor CDVaa para a soma total de aminoácidos em todos os alimentos, com exceção ao GT e ao FTA, iguais aos demais, e ao FTG, que diferiu apenas do tratamento 3. O resultado do GT possivelmente pode ser atribuído à quantidade e qualidade de seu perfil de aminoácidos. 
Avaliação dos coeficientes de digestibilidade...

Tabela 8 Coeficiente de digestibilidade verdadeira (\%) da soma total dos aminoácidos (AA), da soma dos aa não essenciais $\left(\mathrm{AA}_{\mathrm{ne}}\right)$, da soma dos aa essenciais $\left(\mathrm{AA}_{\mathrm{e}}\right)$ e da soma dos aa limitantes (LIM) dos alimentos

\begin{tabular}{|c|c|c|c|c|c|c|c|c|c|}
\hline \multirow{2}{*}{ Aminoácido } & \multirow{2}{*}{ Método } & \multicolumn{8}{|c|}{ Alimento $^{1}$} \\
\hline & & RR & TIM & GT & FTC & FTE & FTH & FTA & FTG \\
\hline \multirow{4}{*}{ AA Totais } & Trat. 1 & $91,34 a$ & $91,17 a$ & $88,23 a$ & $91,22 a$ & $87,67 a$ & $83,63 a$ & $79,17 a$ & $74,84 \mathrm{~b}$ \\
\hline & Trat. 2 & $90,13 a$ & $92,56 a$ & $86,74 a$ & $88,70 a$ & $89,10 a$ & $76,67 \mathrm{c}$ & 77,75ab & $79,58 b$ \\
\hline & Trat. 3 & $91,43 a$ & $85,30 \mathrm{~b}$ & $84,47 a$ & $87,99 a$ & $88,41 a$ & $80,37 b$ & 71,77b & $83,69 a$ \\
\hline & Trat. 4 & $85,10 \mathrm{~b}$ & $78,11 \mathrm{c}$ & $89,35 a$ & $80,70 \mathrm{~b}$ & $79,53 b$ & $69,87 d$ & $75,12 \mathrm{ab}$ & $75,11 b$ \\
\hline \multirow{4}{*}{$\mathrm{AA}_{\mathrm{ne}}$} & Trat. 1 & $90,90 \mathrm{~b}$ & $90,64 a$ & $89,96 a$ & $91,44 a$ & $89,85 a$ & $83,47 a$ & 79,09a & $71,78 \mathrm{~b}$ \\
\hline & Trat. 2 & $89,29 b$ & $92,27 a$ & $88,50 a$ & $88,24 b$ & $88,87 a$ & $73,82 \mathrm{c}$ & $74,92 a$ & $76,37 \mathrm{~b}$ \\
\hline & Trat. 3 & $93,57 a$ & $84,95 b$ & $87,11 a$ & $85,99 b$ & $89,31 a$ & $78,10 \mathrm{~b}$ & $73,37 a$ & $83,05 a$ \\
\hline & Trat. 4 & $84,92 \mathrm{c}$ & $77,53 \mathrm{c}$ & $89.97 a$ & $82,84 \mathrm{c}$ & $83,27 b$ & $71,39 \mathrm{c}$ & $76,84 a$ & $73,36 b$ \\
\hline \multirow{4}{*}{$\mathrm{AA}_{\mathrm{e}}$} & Trat. 1 & $91,67 a$ & $91,57 \mathrm{a}$ & $86,93 a$ & $91,05 a$ & $86,03 a$ & $83,75 a$ & $79,24 a$ & $77,14 b$ \\
\hline & Trat. 2 & $90,76 a$ & $92,76 a$ & $85,41 a$ & $89,05 a$ & $89,27 a$ & $78,80 \mathrm{~b}$ & $79,87 a$ & $81,99 a$ \\
\hline & Trat. 3 & $89,83 a$ & $85,58 b$ & $82,48 a$ & $89,49 a$ & $87,73 a$ & $82,06 a$ & $70,58 b$ & $84,18 \mathrm{a}$ \\
\hline & Trat. 4 & $85,24 b$ & $78,55 \mathrm{c}$ & $88,89 a$ & $79,10 \mathrm{~b}$ & $76,73 b$ & $68,74 \mathrm{c}$ & 73,83ab & $74,92 b$ \\
\hline \multirow{4}{*}{$\mathrm{LIM}^{2}$} & Trat. 1 & $95,67 a$ & $92,50 \mathrm{a}$ & $91,55 a$ & $92,35 a b$ & $89,31 a$ & $88,12 \mathrm{a}$ & $82,81 a$ & $84,27 a$ \\
\hline & Trat. 2 & $93,31 b$ & $92,82 a$ & $89,12 a$ & $90,61 b$ & $92,16 a$ & $85,11 a$ & $86,30 a$ & $87,14 a$ \\
\hline & Trat. 3 & $93,94 a b$ & $90,69 a$ & $88,79 a$ & $94,46 a$ & $90,26 a$ & $86,51 a$ & $73,92 b$ & $84,60 a$ \\
\hline & Trat. 4 & $90,85 c$ & $80,70 \mathrm{~b}$ & $92,19 a$ & $73,14 \mathrm{c}$ & $85,09 \mathrm{~b}$ & $74,86 \mathrm{~b}$ & $80,74 a$ & $81,28 \mathrm{a}$ \\
\hline
\end{tabular}

Valores na mesma coluna, para uma mesma variável, seguidos de letras distintas diferem entre si pelo teste SNK $(\mathrm{P}<0,05)$

1: RR= ração-referência, TIM= trigo integral moído, GT= germe de trigo, FTC= farinha de trigo clara, FTE= farinha de trigo escura, $\mathrm{FTH}$ = farelo de trigo para uso humano, FTA= farelo de trigo para uso animal, FTG= farelo de trigo grosso.

2: Metionina, treonina e lisina.

Na Tab. 9 apresentam-se os valores das perdas metabólicas e endógenas de aminoácidos(mg), expressas por aves em 24 horas. As aves cecectomizadas apresentaram maior excreção de aminoácidos endógenos por ave/dia, com exceção aos valores de prolina, alanina e metionina, que foram iguais àqueles de aves não cecectomizadas. Esse efeito parece ser devido muito mais ao peso das aves do que propriamente à cecectomia. Quando o efeito foi expresso em mg/100 gramas de ave (Tab. 10), essas diferenças não foram significativas, com exceção aos valores de prolina e alanina, que foram maiores, e ao de aspartato, que foi menor nos frangos intactos. Em peso absoluto os valores obtidos nas aves do tratamento 1 foram semelhantes àqueles encontrados nas aves do tratamento 2, com exceção a isoleucina e histidina.

Tabela 9. Perdas endógenas de aminoácidos (mg) expressas por aves em 24 horas

\begin{tabular}{|c|c|c|c|c|c|}
\hline \multirow{2}{*}{ Aminoácido } & \multicolumn{4}{|c|}{ mg/ ave } & \multirow{2}{*}{$\mathrm{CV}$} \\
\hline & Trat. 1 & Trat. 2 & Trat. 3 & Trat. 4 & \\
\hline Aspartato & $13,65 c$ & $16,23 c$ & $26,85 b$ & $56,58 a$ & 12,09 \\
\hline Tirosina & $7,59 c$ & $9,05 c$ & $21,35 b$ & $27,53 a$ & 23,37 \\
\hline Serina & $10,61 \mathrm{c}$ & $12,22 \mathrm{c}$ & $19,94 b$ & $30,36 a$ & 20,02 \\
\hline Glutamina & $22,55 b$ & $24,79 b$ & $34,69 b$ & $60,99 a$ & 23,66 \\
\hline Prolina & $10,52 b$ & $11,94 b$ & $39,78 a$ & $39,60 \mathrm{a}$ & 18,56 \\
\hline Alanina & $10,59 b$ & $10,96 b$ & $26,42 a$ & $24,05 a$ & 29,06 \\
\hline Valina & $8,56 b$ & $8,88 b$ & $14,12 \mathrm{ab}$ & $16,51 \mathrm{a}$ & 27,50 \\
\hline Metionina & $3,14 a$ & $2,55 a$ & 5,90a & $5,97 a$ & 50,27 \\
\hline Isoleucina & $9,26 \mathrm{bc}$ & $14,81 \mathrm{~b}$ & $5,42 c$ & $21,40 \mathrm{a}$ & 33,11 \\
\hline Leucina & $13,06 b$ & $14,53 b$ & $17,29 b$ & $61,03 a$ & 23,33 \\
\hline Treonina & $7,67 \mathrm{c}$ & $7,02 \mathrm{c}$ & $17,87 b$ & $24,12 \mathrm{a}$ & 32,52 \\
\hline Fenilalanina & $13,86 b$ & $11,55 b$ & $16,51 b$ & $36,14 a$ & 22,13 \\
\hline Histidina & $15,46 b$ & $7,74 \mathrm{c}$ & $18,42 \mathrm{ab}$ & $23,00 \mathrm{a}$ & 19,67 \\
\hline Lisina & $11,89 b$ & $10,72 b$ & $14,73 b$ & $24,31 \mathrm{a}$ & 24,82 \\
\hline Totais & $198,33 b c$ & $163,04 \mathrm{c}$ & $281,84 b$ & $456,62 \mathrm{a}$ & 20,90 \\
\hline
\end{tabular}

Valores na mesma linha, para uma mesma variável, seguidos de letras distintas diferem entre si pelo teste $\mathrm{SNK}(\mathrm{P}<0,05)$. 
Tabela 10. Perdas endógenas em aminoácidos (mg)/100 g de peso vivo em 24 horas

\begin{tabular}{|c|c|c|c|c|c|}
\hline \multirow{2}{*}{ Aminoácido } & \multicolumn{4}{|c|}{$\mathrm{mg} / 100 \mathrm{~g}$ PV } & \multirow{2}{*}{$\mathrm{CV}$} \\
\hline & Trat. 1 & Trat. 2 & Trat. 3 & Trat. 4 & \\
\hline Aspartato & $3,03 a$ & $1,15 c$ & $1,36 \mathrm{bc}$ & $1,72 b$ & 14,20 \\
\hline Tirosina & $1,68 a$ & $0,64 \mathrm{c}$ & $1,08 b$ & $0,84 \mathrm{bc}$ & 19,23 \\
\hline Serina & $2,35 a$ & $0,87 b$ & $1,02 b$ & $0,92 b$ & 18,46 \\
\hline Glutamina & $4,99 a$ & $1,77 b$ & $1,77 \mathrm{~b}$ & $1,85 b$ & 21,53 \\
\hline Prolina & $2,33 a$ & $0,85 b$ & $2,01 \mathrm{a}$ & $1,21 b$ & 16,64 \\
\hline Alanina & $2,35 a$ & $0,78 \mathrm{c}$ & $1,33 b$ & $0,73 c$ & 21,84 \\
\hline Valina & $1,90 \mathrm{a}$ & $0,64 b$ & $0,71 b$ & $0,50 \mathrm{~b}$ & 27,89 \\
\hline Metionina & $0,69 a$ & $0,18 b$ & $0,29 b$ & $0,18 b$ & 36,64 \\
\hline Isoleucina & $2,04 a$ & $1,05 b$ & $0,28 c$ & $0,65 b c$ & 45,45 \\
\hline Leucina & $2,89 a$ & $1,03 \mathrm{~b}$ & $0,89 b$ & $1,85 b$ & 21,90 \\
\hline Treonina & $1,70 \mathrm{a}$ & $0,50 \mathrm{~b}$ & $0,90 \mathrm{~b}$ & $0,88 b$ & 25,84 \\
\hline Fenilalanina & $3,07 a$ & $0,82 b$ & $0,84 b$ & $1,10 \mathrm{~b}$ & 20,96 \\
\hline Histidina & $3,42 a$ & $0,55 b$ & $0,93 b$ & $0,70 \mathrm{~b}$ & 17,73 \\
\hline Lisina & $2,63 a$ & $0,76 \mathrm{~b}$ & $0,88 b$ & $0,74 b$ & 17,18 \\
\hline Totais & $43,83 a$ & $11,61 \mathrm{~b}$ & $14,28 \mathrm{~b}$ & $13,86 \mathrm{~b}$ & 46,86 \\
\hline
\end{tabular}

Valores na mesma linha, para uma mesma variável, seguidos de letras distintas diferem entre si pelo teste $\mathrm{SNK}(\mathrm{P}<0,05)$.

Quanto à excreção total de aminoácidos endógenos e metabólicos, as aves do tratamento 1 apresentaram a menor excreção em peso absoluto (mg/ave) para a maioria dos aminoácidos (exceções: metionina, treonina, fenilalanina, histidina e lisina, cujos valores foram maiores em relação ao tratamento 2), e as do tratamento 4, a maior excreção (Tab. 9). Quando a excreção total de aminoácidos foi expressa por mg/100 gramas de PV (Tab. 10), os pintos apresentaram a maior excreção fecal e metabólica total de aminoácidos. Quando expressa em mg/ $\mathrm{P}^{0,75}$ (Tab. 11), a excreção total de serina, glutamina, valina, metionina, fenilalamina, histidina e lisina continuam sendo a maior no tratamento 1.

Em peso metabólico (Tab. 11), os valores excretados de aspartato, treonina, serina e leucina foram maiores nas aves cecectomizadas em relação às não cecectomizadas, entretanto prolina e alanina foram menores. Embora em peso absoluto a excreção endógena e metabólica de aminoácidos tenha relação com o peso, em tamanho metabólico ou em 100 gramas de PV pode-se notar que os pintos apresentam maior excreção para todos os aminoácidos devido ao maior metabolismo protéico e às altas taxas de reciclagem da proteína corporal. Proporcionalmente, em aves jovens as vísceras representam a maior porcentagem do peso corporal, com alta reciclagem protéica.

Tabela 11. Valores das perdas endógenas em aminoácidos (mg) expressas por idade de peso metabólico em 24 horas

\begin{tabular}{|c|c|c|c|c|c|}
\hline \multirow{2}{*}{ Aminoácido } & \multicolumn{4}{|c|}{$\mathrm{mg} / \mathrm{kg} \mathrm{P}^{0,75}$} & \multirow{2}{*}{$\mathrm{CV}$} \\
\hline & Trat. 1 & Trat. 2 & Trat. 3 & Trat. 4 & \\
\hline Aspartato & $24,92 a$ & $12,58 b$ & $16,13 b$ & 23,16a & 12,90 \\
\hline Tirosina & $13,85 a$ & $7,02 \mathrm{c}$ & $8,72 \mathrm{bc}$ & $11,26 a b$ & 19,04 \\
\hline Serina & $19,36 a$ & $9,47 \mathrm{bc}$ & $7,89 \mathrm{c}$ & $12,42 b$ & 18,25 \\
\hline Glutamina & $41,09 a$ & $19,22 b$ & $20,94 b$ & $24,95 b$ & 22,68 \\
\hline Prolina & $19,21 b$ & $9,26 c$ & $23,84 a$ & $16,20 b$ & 17,14 \\
\hline Alanina & $19,30 \mathrm{a}$ & $8,49 b$ & $15,80 \mathrm{a}$ & $9,84 \mathrm{~b}$ & 24,21 \\
\hline Valina & $15,66 a$ & $6,88 b$ & $8,44 b$ & $6,75 b$ & 27,59 \\
\hline Metionina & $5,72 \mathrm{a}$ & 1,98b & $3,51 b$ & $2,44 b$ & 40,39 \\
\hline Isoleucina & $16,80 \mathrm{a}$ & $11,48 a b$ & $3,29 c$ & $8,75 \mathrm{bc}$ & 41,52 \\
\hline Leucina & $23,82 a$ & $11,26 c$ & $10,48 \mathrm{c}$ & $24,96 a$ & 23,11 \\
\hline Treonina & $14,00 \mathrm{a}$ & $5,44 b$ & $10,66 a$ & $11,91 \mathrm{a}$ & 28,57 \\
\hline Fenilalanina & $25,30 a$ & $8,96 b$ & $9,97 \mathrm{~b}$ & $14,78 b$ & 21,80 \\
\hline Histidina & $28,17 \mathrm{a}$ & $6,00 \mathrm{c}$ & $11,03 b$ & $9,41 \mathrm{bc}$ & 18,25 \\
\hline Lisina & 21,69a & $8,31 b$ & $10,37 b$ & 9,94b & 18,07 \\
\hline Totais & $289,92 a$ & $126,39 c$ & $161,11 \mathrm{bc}$ & $186,78 b$ & 14,57 \\
\hline
\end{tabular}

Por último, uma vez que as perdas metabólicas e endógenas são maiores nas aves cecectomizadas devido ao peso, poder-se-ia conjeturar que essas perdas interfeririam nos resultados de aminoácidos digestíveis. 
Essa posição é absolutamente verdadeira para a CDAaa, uma vez que essa medida é obtida sem levar em consideração as perdas metabólicas e endógenas. Como as aves mais pesadas tiveram as maiores perdas, em valores absolutos, elas apresentaram os menores valores de CDAaa. Nesse caso os menores valores de CDAaa obtidos nas aves cecectomizadas não podem ser atribuídos à ausência de ceco, uma vez que o peso tornou-se um fator de confundimento.

Para a CDVaa, as perdas metabólicas e endógenas são descontadas e, teoricamente, o peso já não é um fator de confundimento. Isso explica porque o grupo em jejum apresentou peso e características idênticas ao restante dos tratamentos experimentais. Nesse caso, quando se subtraíram as perdas endógenas e metabólicas obtidas do grupo em jejum eliminou-se o possível efeito dos pesos distintos entre os tratamentos. Logo, teoricamente, todas as diferenças devem-se somente à cecectomia. Dessa forma, é importante que o grupo em jejum seja homogêneo entre si e entre os demais tratamentos, como proposto por Sibbald (1976b).

\section{CONCLUSÕES}

Nas condições em que se realizou este trabalho pode-se concluir que: 1- os tratamentos com metodologia tradicional apresentam maiores valores de CDAaa quando comparados aos métodos de alimentação forçada; 2- em alguns alimentos os valores de digestibilidade aparente dos aminoácidos são negativos (TRE, ASP, TIR e ALA da FTC e LEU e TRE da FTE, no tratamento 4, com aves cectomizadas) mostrando que a perda endógena é maior do que a ingestão de aminoácidos; 3- a digestibilidade aparente dos aminoácidos é afetada pela metodologia, e a metodologia de alimentação forçada apresenta valores muito baixos ou negativos; 4- as aves cecectomizadas apresentam valores de digestibilidade verdadeira dos aminoácidos inferiores aos das aves normais; 5 - as perdas endógenas totais de aminoácidos em $\mathrm{mg}$ /ave foram diferentes entre todos os tratamentos, sendo afetadas pelo peso das aves; 6- as perdas endógenas totais de aminoácidos em $\mathrm{mg} / 100 \mathrm{~g}$ de $\mathrm{PV}$ e em $\mathrm{mg} / \mathrm{P}^{0,75}$ foram maiores para pintos quando comparadas a todos os outros tratamentos com frangos; 7- as perdas endógenas totais de aminoácidos em $\mathrm{mg} / 100 \mathrm{~g}$ de $\mathrm{PV}$ e em $\mathrm{mg} / \mathrm{P}^{0,75}$ foram semelhante para aves intactas e cecectomizadas.

\section{REFERÊNCIAS BIBLIOGRÁFICAS}

ALBINO, L.T.F. Sistemas de avaliação nutricional de alimentos e suas aplicações na formulação de rações para frangos de corte. 1991. 141p. Tese (Doutorado). Universidade Federal de Viçosa, Viçosa, MG.

BELLAVER, C. Predição da digestibilidade de aminoácidos in vivo com suínos. In: SIMPÓSIO LATINO-AMERICANO DE NUTRIÇÃO ANIMAL E SEMINÁRIO SOBRE TECNOLOGIA DE PRODUÇÃO DE RAÇÕES, Campinas. Anais..., Campinas, 1996, p.171-205.

BORGES, F.M.O.; ROSTAGNO, H.S.; SAAD, C.E.P. et al. Comparação de métodos de avaliação dos valores energéticos do grão de trigo e seus subprodutos para frangos de corte. Arq. Bras. Med. Vet. Zootec., v.55, p.710-721, 2003.

BRAGG, D.B.; IVY, C.A.; STEPHENSON, E.L. Methods for determining amino acid availability of feeds. Poult. Sci., v.47, p.21352137, 1970.

CRISSEY, S.D.; THOMAS, O.P. Comparison of the sensitivities of growth and digestibility studies using intact, cecoctomized, and cannulated roosters. Poult. Sci., v.66, p.866-874, 1987.

ENGSTER, H.M.; CAVE, N.A.; LIKUSKI, H. et al. A collaborative study to evaluate a precision-fed rooster assay for true amino acid availability in feed ingredients. Poult. Sci., v.64, p.487-498, 1985.

HAN, Y.; PARSONS, C.M. Determination of available amino acids and energy in alfalfa meal, feather meal, and poultry by product meal by various methods. Poult. Sci., v.69, p.1544-1552, 1990.

JOHNS, D.C.; LOW, C.K.; JAMES, K.A.C. Comparison of amino acid digestibility using the ileal digesta from growing chickens and cannulateds adult cockerels. Br. Poult. Sci., v.27, p.679-685, 1986a.

JOHNS, D.C.; LOW, C.K.; SEDCOLE, J.R. et al. Determination of amino acid digestibility using caecectomised and intact adult cockerels. Br. Poult. Sci., v.27, p.451-461, 1986b. 


\section{Borges et al.}

KESSLER, J.W.; NGUYEN, T.H.; THOMAS, O.P. The amino acid excretion values in intact and cecoctomized negative control roosters used for determining metabolic plus endogenous urinary losses. Poult. Sci., v.60, p.1576-1577, 1981.

LEE, K.H.; QI, G.H.; SIM, J.S. Metabolizable energy and amino acid availability of full-fat seeds, meals, and oils of flax and canola. Poult. Sci., v.74, p.1341-1348, 1995.

LIKUSKI, H.J.A.; DORRELL, H.G. A bioassay for rapid determinations of amino acid availability values. Poult. Sci., v.57, p.16581660, 1978.

MEJÍA, A.M.G.; FERREIRA, W.M. Métodos de avaliação da disponibilidade da proteína e dos aminoácidos nos alimentos para não ruminantes. In: SIMPÓSIO INTERNACIONAL DE PRODUÇÃO DE MONOGÁSTRICOS, 1, Seropédica, RJ. Anais...,Seropédica, 1996.

MOTTER, J.H. Fundamental principies of HPLC. s.l.: Shimadzu, 1991. 49p

MUZTAR, A.J.; SLINGER, S.J. Bioavailable amino acids in corn and alfafa as measured by applying the true metabolizable energy assay. Poult. Sci., v.59, p.1873-1877, 1980.

PUPA, J.M.R.; LEÃO, M.I.; CARVALHO, A.U. et al. Cecectomia em galos sob anestesia local e incisão abdominal. Arq. Bras. Med. Vet. Zootec., v.50, p.531-535,1998.

ROSTAGNO, H.S.; SILVA, D.J.; COSTA, P.M.A. et al. Composição de alimentos e exigências nutricionais de aves e suínos. Viçosa: Imprensa Universitária, 19.ed., 1985. 59p.

SIBBALD, I.R. A bioassay for true metabolizable energy in feedingstuffs. Poult. Sci., v.55, p.303-308, 1976.

SIBBALD, I.R. Estimation of bioavailable amino acids in feedingstuffs for poultry and pigs: a review with emphasis on balance experiments. Can. J. Anim. Sci., v.67, p.221-300, 1987. 